\title{
A radar echo signal detection algorithm in low signal-to-noise ratio
}

\author{
Xiangju Li ${ }^{1, a}$ \\ Information Engineering College, Dalian University, Dalian116622, China; \\ a1031944301@qq.com
}

Keywords: Fractional Fourier transform, LFM, Signal detection, SNR

\begin{abstract}
According to the problem of radar echo signal detection in the low signal-to-noise rate, an improved detection algorithm of fractional Fourier transform detect signal after wavelet filtering is proposed. Because of the low detection performance of fractional Fourier transform in the low signal-to-noise rate as well as considering the non-stationary and time-varying features of the radar echo signals, wavelet denosing method is introduced to preprocess echo signal and improve the radar echo signals signal-to-noise rate. Then Fractional Fourier Transform detected the target preferably. Fractional Fourier Transform has special accumulating quality for LFM signal. The results of the simulations show that corresponding algorithm proposed in this article is superior to the fractional Fourier transform algorithm.
\end{abstract}

\section{Introduction}

Fractional Fourier transform can be interpreted as the method that signal rotates around the origin counterclockwise at any angel in the time-frequency plane and then constructs a fractional Fourier domain ${ }^{[1]}$. Because the fractional Fourier transform is just using accumulating quality for LFM signal, it is not suppress the noise. So when the target echo signal is weak, that is to say the signal-to-noise ratio is low, detection performance of the fractional Fourier transform will decline ${ }^{[2]}$. To solve this problem, this paper proposes a new method. Wavelet denosing method is introduced to preprocess echo signal and improve the radar echo signals signal-to-noise rate, then the weak target signal is detected by the fractional Fourier transform. That is, Fractional Fourier transform detect radar echo signals in combination with wavelet analysis.

\section{Algorithm Principle}

\subsection{Fractional Fourier transform}

Fractional Fourier transform is also referred to as the angel Fourier transform, the Fractional Fourier transform of the function $x(t)$ can be represented as $X_{P}(u)$ or $F^{P} x(u)$, its definition is ${ }^{[3]}$ :

$$
X_{P}(u)=\int_{-\infty}^{+\infty} K_{P}(t, u) x(t) d t
$$

where $p$ is the order and can take arbitrary real number. $\alpha=p \pi / 2$, and it is the rotation angle, $n$ is integer.

Transform nuclear $K_{P}(t, u)$ of FRFT is:

$$
K_{P}(t, u)=\left\{\begin{array}{l}
\sqrt{\frac{1-j \cot \alpha}{2 \pi}} e^{j \frac{t^{2}+u^{2}}{2} \cot \alpha-j t u \csc \alpha}, \alpha \neq n \pi \\
\delta(t-u), \alpha=2 n \pi \\
\delta(t+u), \alpha=(2 n \pm 1) \pi
\end{array}\right.
$$

Where $n$ is an integer.

Fractional Fourier transform can be seen that a signal can be decompose to a complete orthogonal basis in fractional Fourier domain. It can get the basis of different chirp rate by changing the rotating angle, to make the signal achieve the best energy concentration characteristics on a 
basis, thus detecting the target signal preferably ${ }^{[4]}$. Literature[5] has a very detailed analysis of Fractional Fourier transform to deal with the principle of linear frequency modulation signal from two aspects. That is the kernel function of FRFT and the time-frequency distribution.

\section{2 wavelet denoising}

Many method of wavelet denoising have be put forward.Among them, the method of modulus maxima denoising is more commonly used. It is put forward by the Mallet ${ }^{[6]}$. Take a point $\left(a_{0}, b_{0}\right)$ in $W_{f}(a, b)$, to make $b$ belongs to $b_{0}$ neighborhood, if

$$
\left|W_{f}\left(a_{0}, b\right)\right| \leq\left|W_{f}\left(a_{0}, b_{0}\right)\right|
$$

set up, then $W_{f}\left(a_{0}, b_{0}\right)$ is called the modulus maxima of wavelet coefficients on the scale $a_{0}$. The modulus maxima of wavelet coefficients is produced by singular points and noise of the signal. The law of the amplitude versus the scale depends on the Lipschitz exponent ${ }^{[7]}$ of the signal singularity, that is:

$$
\left|W_{f}\left(a_{0}, b\right)\right| \leq k a_{0}{ }^{L}
$$

Where $k$ is a constant. From the above formula: When $L$ is greater than $\operatorname{zero}(L>0)$, the modulus maxima increases with the increase of the value of $a$; When $L$ is less than zero $(L<0)$, the modulus maxima reduces with the increase of the value of $a$. From the literature [7], $L>0$ is for the general signal and $L \leq 0$ for the noise conversely. As a result, we can distinguish signal and white noise on the modulus maxima phase-plane of wavelet coefficients. Nosie can be wipped off by removing the mudulus maxima of wavelet coeffients corresponding to noise and preserving the mudulus maxima of the signal ${ }^{[8]}$.

\section{Detection algorithm}

Radar echo includes useful signal and clutter. According to impact of clutter on signal detection, clutter is approximate to additive noise. Therefore, the received signal can be viewed as target signal and Gaussian white noise. Expression of the model is:

$$
x(t)=s(t)+n(t)
$$

Among them, $s(t)$ is the target signal, $n(t)$ is the Gaussian white noise. $x(t)$ is the Radar echo signal.

Detection process:Firstly, we need to preprocess echo signal by wavelet filtering. Secondly, with the rotation order $p$ as the variable, apply the Fractional Fourier transform over the reconstruction echo signal to form a 2D distribution of the signal energy on the coordinate plane $(p, u)$. The target signal can be detected by the two-dimensional search for the peak point according to the threshold on the plane.

In this paper, it takes the wavelet transform modulus maxima method to remove the noise. First of all, the echo signal was carried out binary discrete wavelet transform. In order to make the signal energy is superior to the noise energy on the largest scale, it should the make wavelet coefficients of signal focus on this scale. We generally choose 4 : 5 for the wavelet decomposition scale, this article choose $j=4$, and use the db5 as wavelet basis function. Then, starting with the largest scale, we select the mudulus maxima of the signal wavelet coefficients by scale. we choose hard threshold method on the largest scale. In the low signal-to-noise ratio, and the wavelet coefficients of signal is focused on the largest scale, we can use the amplitude of the wavelet coefficients of signal to determine the threshold. That is $t h=c M / j, M$ is the maximum amplitude on the largest scale $2^{j}$, $c$ is constant.After we determine all the wavelet transform modulus maxima on the largest scale. According to the corresponding positional relation, we find modulus maxima of the next scale. According to the amplitude relationship, we find the nature of the modulus maxima. Then, we retain the modulus maxima of signal and remove the modulus maxima of noise. We determine the one level of modulus maxima. Then, according to the recursive construction method we select the 
modulus maxima of next scale. According to Mallat's reconstruction algorithm, we use reserved wavelet coefficients to reconstruct the signal.

Noise of Radar echo signal is removed after wavelet filtering, signal-to-noise ratio has been improved. Then, the reconstructed signal is detected by Fractional Fourier Transform, from the time domain to the Fractional domain. Echo signal is detected on the Fractional domain, searching range of fractional order $p$ is set to $[0,2]$. In order to reduce the amount of calculation and running time, at the same time it can get higher estimation accuracy of the order $p$. So we adopt two steps. We set a large search step at the first scan search, and we can get the approximate range of the order of $p$. Then, we set a smaller search step in a small range that we have searched. So we obtain an accurate value of the order of $p$. Thus we detect the target.

\section{Simulation experiment and performance analysis}

Simulation conditions: Background noise is Gaussian white noise. The model of Target echo is:

$s(t)=\exp \left[j 2 \pi\left(f_{0} t+\frac{1}{2} k t^{2}\right)\right]$

Parameter selection, center frequency $f_{0}=150 \mathrm{MHz}$, sampling frequency $f_{s}=512 \mathrm{MHz}$ and sampling points $N=1024$, chirp rate $k=30 \times 10^{12} \mathrm{HZ} / \mathrm{S}$, Time-domain waveform of echo signal (including noise) is illustrated by the Figure. 1.

Signal is detected by Fractional Fourier Transform. In order to reduce the amount of calculation and running time, at the same time it can get higher estimation accuracy of the order $p$. The search step length is set 0.01 by a large number of experimental results. Then we obtain a three-dimensional figure on fractional domain. We can see that value of the order $p$ is about from 0.90 to 1.20 . Then we search again in this range and the search step length is set 0.001 . We get more precise value of the order $p$, it is 1.075. As shown in the figure. 2:
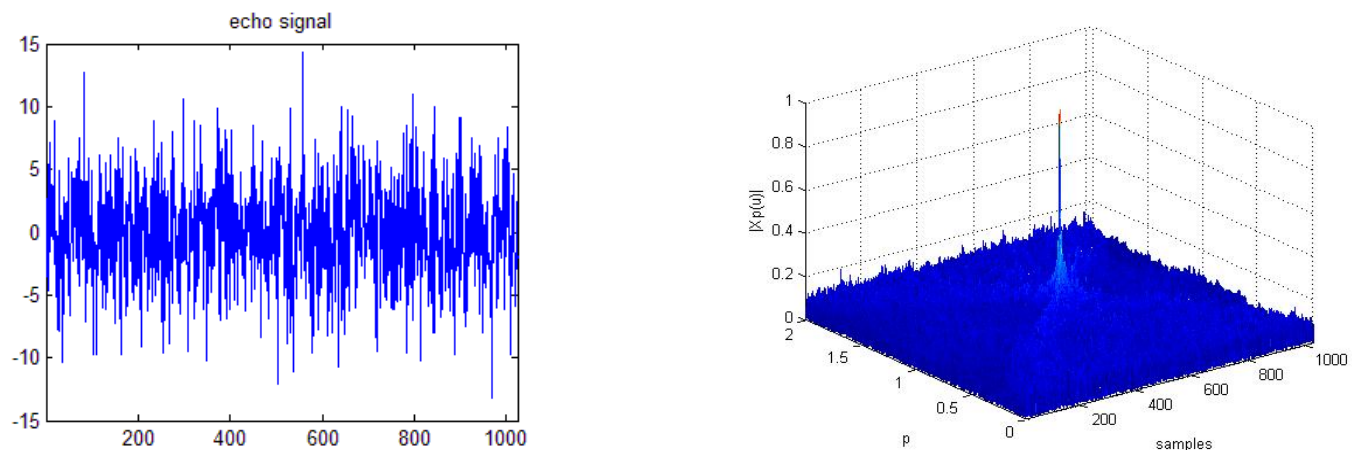

Fig. 1 The echo signal

Fig. 2 The three-dimensional figure on the plane of $(p, u)$

Then Fractional Fourier transform detect echo signals on the optimal order and LFM signal can be detected. When signal-to-noise ratio (SNR) is high, two algorithm can both detect the target echo signal.

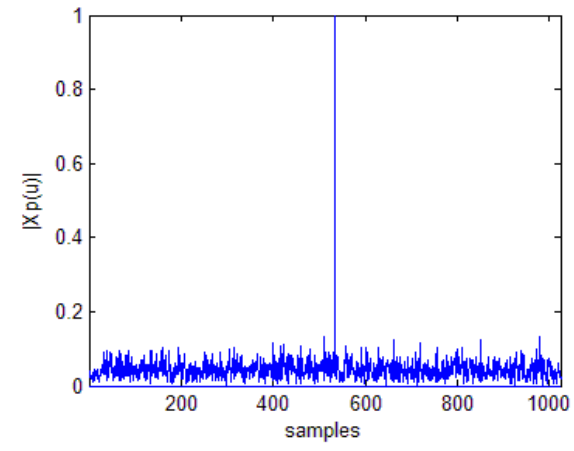

Fig. 3 The detection results of FRFT algorithm

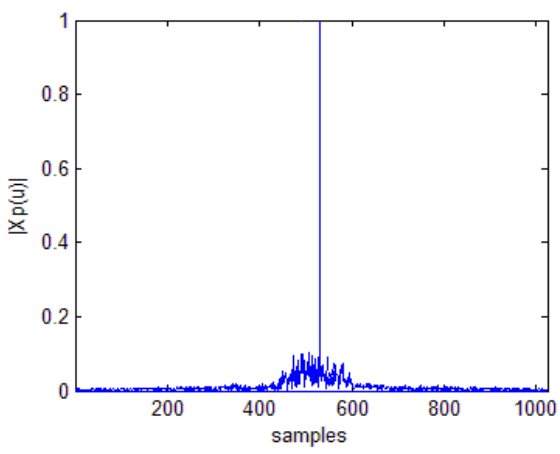

Fig. 4 The detection results of FRFT 
after wavelet filtering

When the signal-to-noise ratio is low, the simulation results are shown in Figure 4 and Figure 5. That is, algorithms of FRFT is almost undetectable target, while corresponding algorithm proposed in this article can still detect target.

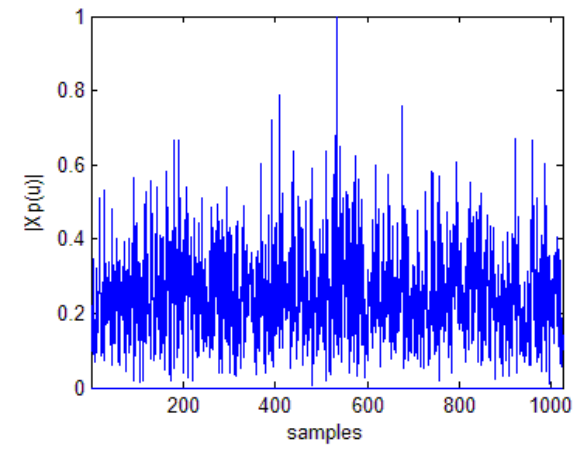

Fig. 5 The detection results of FRFT algorithm

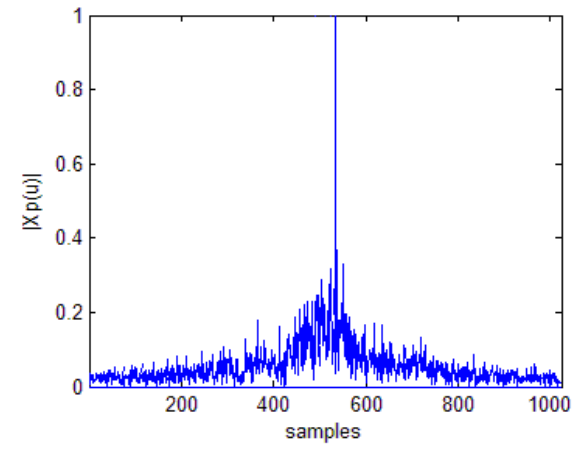

Fig. 6 The detection results of FRFT Fig. 6 1

\section{Conclusion}

The fractional Fourier transform is an effective detection method for non-stationary signal. In this paper, it joined the wavelet denoising link on the basis of the FRFT and improved the signal-to-noise ratio of echo signal. At this time, echo signal is detected by FRFT and detection performance can be improved. That is to say, when the SNR is low, corresponding algorithm proposed in this article is superior to the fractional Fourier transform algorithm. In fact, this article is a combination of advantages of both single method and construct a new target detection method. The detection process is more complicated than a single method. Although the detection performance has be improved, but it increase the amount of computation. Therefore it needs to be selected according to the actual needs of the project implementation.

\section{References}

[1]. Chen Xiaolong, Liu Ningbo, Wang Guoqing, Guan Jian. Radar Detection Method for Moving Target Based on Radon-Fourier Fractional Fourier Transform[J]. Acta Electronica Sinica. Vol. 42 (2014) No. 6, p. 1074-1080.

[2]. Hu Guangbo, He Xibing, Gan Xinnian. Target detection algorithm based on Fractional Fourier Transform cumulant [J]. Torpedo technology.Vol. 19 (2011) No. 5, p. 344-348.

[3]. Tao Ran, Qi Lin, Wang Yue. Principles and Applications of Fractional Fourier Transform[M]. Tsinghua University press, 2004, p.55-78.

[4]. Liang Hong, Liu Jinbo. A method of high speed moving target detection in reverberation[J]. Torpedo technology. Vol. 17 (2009) No. 5, p. 44-47.

[5]. Deng Yi, Liang Yan, Ling Jiping. Linear frequency-modulated signal detection algorithm based on the fractional Fourier transform[J]. Ship Electronic Engineering. Vol. 31 (2011) No. 9 ,p. 43-46.

[6]. Qiushi Hao, Yi Shen, Yan Wang, Xin Zhang. De-noising of rail crack AE signal based on wavelet modulus maxima. Instrumentation and Measurement Technology Conference(I2MTC). Italy, 2015(5), p.675-680.

[7]. Feng Ganzhong, He Zhiming. Wideband Radar signal denoising method based on modulus maximum[J]. Vol. 35 (2013) No.6, p.38-41.

[8]. Li Longyun, Peng Yuhua. An denoising method for radar signals based on wavelet transform 
modulus maxima[J]. Journal of Electronic Measurement and Instrument, Vol. 18 (2004) No. 3, p. 76-79. 